\title{
Stafne Bone Cavity of the Mandible
}

Jae Il Lee,

Seok Joo Kang,

Seong Pin Jeon, Hook Sun

Department of Plastic and Reconstructive Surgery, Busan Baik Hospital, Inje University School of Medicine, Busan, Korea

No potential conflict of interest relevant to this article was reported.
Stafne bone cavity is a rare mandibular defect that was first reported by Edward C. Stafne in 1942. It commonly presents with a well-demarcated, asymptomatic, unilateral radiolucency that indicates lingual invagination of the cortical bone. A 52-year-old female patient who with nasal bone fracture, visited the hospital. During facial bone computed tomography (CT) for facial area evaluation, a well-shaped cystic lesion was accidentally detected on the right side of the mandible. Compared to the left side, no swelling or deformity was observed in the right side of the oral lesion, and no signs of deformity caused by mucosal inflammation. 3D CT scans, and mandible series x-rays were performed, which showed a well-ossified radiolucent oval lesion. Axial CT image revealed a cortical defect containing soft tissue lesion, which has similar density as the submandibular gland on the lingual surface of the mandible. The fact that Stafne cavity is completely surrounded by the bone is the evidence to support the hypothesis that embryonic salivary gland is entrapped by the bone. In most cases, Stafne bone cavity does not require surgical treatment. We believe that the mechanical pressure from the salivary gland could have caused the defect.

Keywords: Bone cyst / Mandible / Image / Three-dimensional

\section{INTRODUCTION}

Stafne bone cavity (SBC) is a rare mandibular defect that was first reported by Stafne in 1942 [1]. It commonly presents as a well-demarcated, asymptomatic, unilateral radiolucency that indicates lingual invagination of the cortical bone. These lesions are usually discovered by chance, mostly through conventional radiological examinations, and are often erroneously identified as traumatic lesions or mass lesions of the chin [2].

The authors report a case of SBC and discuss the causes, clinical and radiographic characteristics, and differential diagnoses of this rare condition.

This study was reviewed and approved by the Ethics Review Board of the InJe University Health Center.

\section{Correspondence: Seok Joo Kang}

Department of Plastic and Reconstructive Surgery, Busan Baik Hospital, Inje University School of Medicine, 75 Bokji-ro, Busanjin-gu, Busan 47392, Korea

E-mail: sonydr@naver.com

Received March 21, 2016 / Revised June 8, 2016 / Accepted June 9, 2016

\section{CASE REPORT}

A 52-year-old female patient presented with facial injury suspicious for nasal bone fracture. During facial bone computed tomography (CT), a well-circumscribed cystic lesion was discovered incidentally on right side of the mandible (Fig. 1). The patient did not complain of any discomfort, pain, or numbness of the lower face. She also denied any trauma to the chin or significant surgical history.

Lateral radiographs showed a well-defined, radiolucent, ovoid lesion anterior to the right mandibular angle (Fig. 2). The coronal and sagittal CT images demonstrated a deficiency in the mandibular defect containing the soft tissue lesion in continuity with the submandibular gland (Fig. 3). Based on the radiological findings, the patient was diagnosed with a Stafne bone cavity, and regular observation was recommended. 


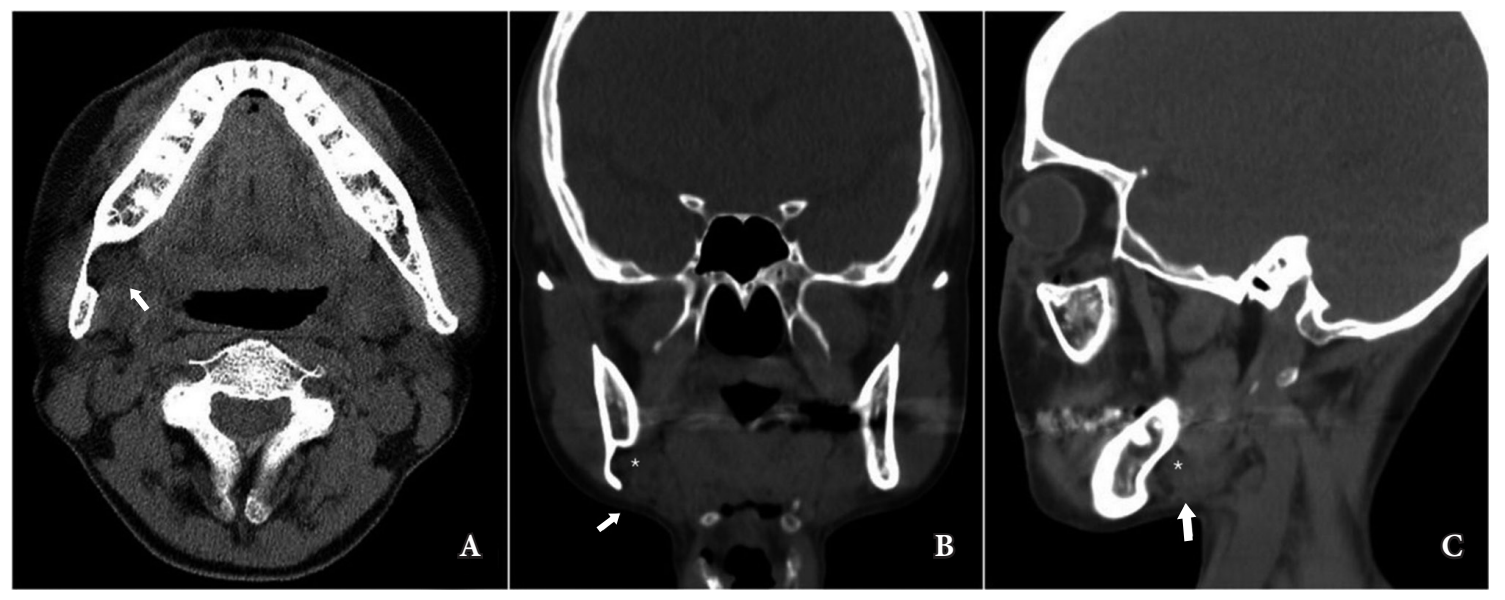

Fig. 1. Incidental finding of bony cavity in the mandible. Axial (A) image reveals the radiolucent lesion (arrow) as a cortical defect containing soft a tissue lesion that has similar density with submandibular gland, on the lingual surface of the mandible. Coronal (B) and sagittal (C) images demonstrate the deficit of the mandibular defect containing the soft tissue lesion (asterisk) that is continuous with the submandibular gland (arrow).

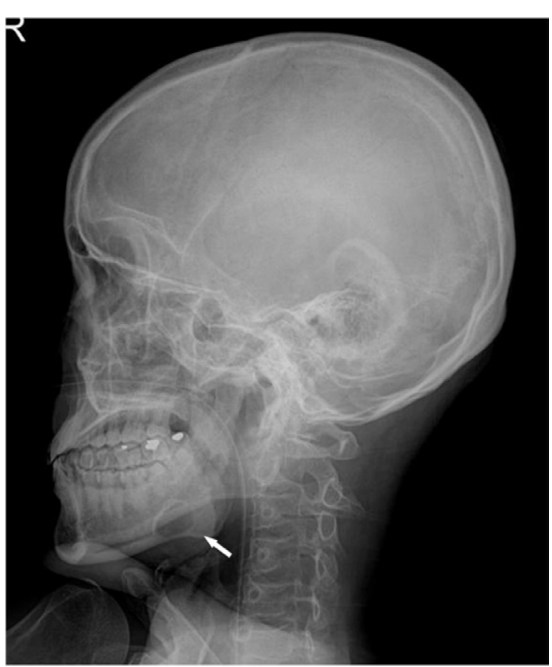

Fig. 2. Lateral radiograph shows a well-defined, radiolucent, ovoid lesion anterior to the right mandibular angle (arrow).

\section{DISCUSSION}

After the report by Stafne, others have described similar cavities that were often observed in men between 50 to 70 years of age, with $0.10 \%-0.48 \%$ prevalence [3]. SBC is easily diagnosed by the presence of a cyst-like oval radiolucency near the gonial angle of mandible on panoramic radiography. Even though these lesions are benign, they can be mistaken for bone metastasis or other common cysts. Thus, cross-sectional imaging studies, such as

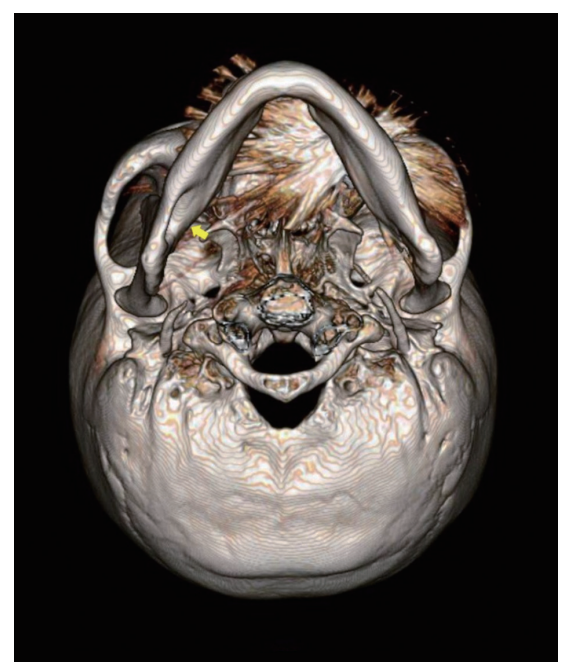

Fig. 3. Three-dimensional computed tomography image reveals a bony defect on the lingual surface of the mandible (arrow).

cone-beam CT (CBCT) or magnetic resonance imaging (MRI), is useful. CBCT is non-invasive and effective for examining bone boundaries. MRI provides clearly defined tissue borders and allows for the examination of soft tissues. Sialography is an option for lesions in the salivary gland, although its use is restricted because of the small diameter of the Rivinus ducts.

Diseases that must be diagnosed differently from Stafne bone cavity are cysts, including odontogenic cyst, traumatic bone cyst, periapical cyst, and residual cyst. Moreover, it must be dif- 
ferentiated from tumors such as ameloblastoma, giant cell tumor, and brown tumor, as well as nonossifying fibroma, vascular malformation, and fibrous dysplasia [3].

The histopathology of SBC has reported absence of any cystic lesion but rather the presence of a mixed salivary gland tissue, consistent with normal sublingual gland [3].

In most cases, Stafne bone cavity does not require surgical treatment. In rare cases where persistent infection or malignant changes occur, biopsy or surgical exploration must be performed [4].

The pathogenesis of this lingual bone cavity is not yet fully understood [5]. Many authors, including Stafne, have suggested that the condition could arise due to a congenital defect leading to the entrapment of a portion of glandular tissue during mandibular development and ossification. This theory was supported by the existence of three salivary glands in the region, which are seen on radiographs as radiolucent structures [2,3,5]. Based on many reports, SBC is believed to be an ectopic portion of the submandibular salivary gland, which causes remodeling of the lingual cortex, thereby resulting in the bone defect.

In our case, axial CT image revealed a cortical defect containing soft tissue, which had density similar to that of the submandibular gland on the lingual surface of the mandible. The fact that SBC is completely surrounded by the bone is the evidence to support the hypothesis that embryonic salivary gland is entrapped by the bone.

In the case of the SBC discovered in a 52-year-old female patient, we believe that the mechanical pressure from the salivary gland could have caused the defect, which contributed to the pathophysiology of future research SBC. Stafne bone cavities are mostly asymptomatic, and the posterior SBC can be easily diagnosed through radiography. However, a surgeon managing a patient with SBC must be fully knowledgeable about the disease and needs to be capable of differentiating SBC from other potential pathologic conditions. It is important to avoid unnecessary examinations and surgical procedures in these patients.

\section{REFERENCES}

1. Stafne EC. Bone cavities situated near the angle of the mandible. J Am Dent Assoc 1942;29:1969-72.

2. Turkoglu K, Orhan K. Stafne bone cavity in the anterior mandible. J Craniofac Surg 2010;21:1769-75.

3. Taysi M, Ozden C, Cankaya B, Olgac V, Yildirim S. Stafne bone defect in the anterior mandible. Dentomaxillofac Radiol 2014;43:20140075.

4. Venkatesh E. Stafne bone cavity and cone-beam computed tomography: a report of two cases. J Korean Assoc Oral Maxillofac Surg 2015;41:145-8.

5. Shimizu M, Osa N, Okamura K, Yoshiura K. CT analysis of the Stafne's bone defects of the mandible. Dentomaxillofac Radiol 2006;35:95102. 